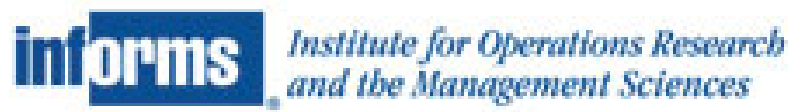

The Direct Solution of the Transportation Problem with Reduced Matrices

Author(s): Paul S. Dwyer

Source: Management Science, Vol. 13, No. 1, Series A, Sciences (Sep., 1966), pp. 77-96

Published by: INFORMS

Stable URL: http://www.jstor.org/stable/2627913

Accessed: 12/10/2011 20:37

Your use of the JSTOR archive indicates your acceptance of the Terms \& Conditions of Use, available at http://www.jstor.org/page/info/about/policies/terms.jsp

JSTOR is a not-for-profit service that helps scholars, researchers, and students discover, use, and build upon a wide range of content in a trusted digital archive. We use information technology and tools to increase productivity and facilitate new forms of scholarship. For more information about JSTOR, please contact support@ jstor.org. 


\title{
THE DIRECT SOLUTION OF THE TRANSPORTATION PROBLEM WITH REDUCED MATRICES*
}

\author{
PAUL S. DWYER ${ }^{1}$
}

\author{
The University of Michigan
}

\begin{abstract}
A discussion of the importance of a direct method in obtaining all the solutions of a transportation problem, and in obtaining solutions of more general problems, is followed by a discussion of methods of reduced matrices in which the transportation matrix is reduced, by a series of subtractions from rows and columns, to a transformed matrix to which the orthogonality condition is applicable. The direct method proceeds in a series of simple steps to the determination of zero terms having associated $x_{i j}$ values which eventually satisfy the row and column equations. Formal and informal versions are presented and application is made to several general problems.
\end{abstract}

\section{Introduction}

We seek methods which produce all the solutions of a transportation problem and which are applicable to more complex transportation problems. It is argued that direct methods should be considered. While the purest direct method is not practical, a modification substitutes some simple techniques for the minimization condition. With the methods of reduced matrices, we make subtractions from the rows and columns of the transportation matrix to produce a transformed matrix with all elements non-negative such that the non-negative integral $x_{i j}$ can be assigned to the zero terms so as to satisfy the specifications for origins, $i$, and destinations, $j$.

This work is a revised and extended version of an unpublished paper of 1955 [6]. It is related to published work on (and machines programs for) more general problems published in 1956 [7] and 1957 [9] and [17].

\section{The Problem}

The transportation matrix, $C=\left\|c_{i j}\right\|$, is an $m x n$ matrix with associated integral $x_{i j} \geqq 0$ to be determined according to the specifications

$$
\sum_{i=1}^{m} x_{i j}=b_{j} ; \quad \sum_{j=1}^{n} x_{i j}=a_{i} ; \quad \sum_{i=1}^{m} a_{i}=\sum_{j=1}^{n} b_{j}=N
$$

such that

$$
T=\sum_{i j} x_{i j} c_{i j} \text { is as small as possible. }
$$

\section{General Solutions and Solutions of More General Problems}

For most purposes it would seem that an important property of a proposed method for solving a problem is that it produces all the solutions, and not just one of them. For some purposes it may even be desirable that the solution may

* Received August 1965 and revised April 1966.

${ }_{1}^{1}$ Research supported in part by National Science Foundation Grant GP-4308. 
include solution sets which are excluded from the usual statement of the problem. Thus the solution of the transportation problem and particularly the solution of the solid or $k$-dimensional problem [9], might well be given in terms of the more general solutions resulting from the use of fractional as well as integral assignments. It is also very desirable that a method be applicable, with no more than slight modification, to various generalizations of the problem. Thus it is desirable that a method for solving the transportation problem be also essentially applicable to the $k$-dimensional transportation problem [9], to transportation problems with upper bounds [5, 368], to transportation problems with bounded partial sums of variables [15], to fixed charge transportation problems with constant fixed charges [22] and to problems having the same $c_{i j}$ but with different values of $a_{i}$ and $b_{j}$ [3].

The attempt here is to provide a general method which gives general solutions to the general problems mentioned above which is operationally practical and generally more efficient than rival methods.

\section{Direct Methods}

Frequently a direct method of solving a problem can be extended to direct generalizations of the problem. By a direct method we mean one in which the basic specifications of the problem are used directly in solving the problem without replacing them, in whole or in part, with auxiliary theorems or criteria and without using the circuitous approach of transforming an initial feasible solution to an optimal one. In minimization (maximization) combinatorial problems such as the transportation problem, the purest direct method consists in writing out all possible feasible solutions which satisfy (1) and selecting those which satisfy (2). This method gives a general answer to the problem, in a sense that a circuitous method does not, and since it is direct it is applicable to many direct generalizations of the problem which may result from additional specifications which are similar in form to those of (1). Generally the more direct methods are subject to more immediate direct generalization since direct methods do not depend on theorems or criteria which, while they are the complete equivalent of the conditions of the original problem, are commonly inapplicable to the more complex ones. Other things being equal, we seek methods which are direct if they are to be applicable to direct generalizations of the original problem.

But other things are not always equal! The pure direct method of writing out all possible feasible solutions - those which satisfy (1) - is simply not practical in most problems. In practice we must resort to methods which are, in part at least, indirect. The important point is that, if we wish to have methods which are applicable to direct generalizations of the problem, we should attempt to use a minimum of equivalence theorems and auxiliary criteria as substitutes for the stated conditions of the problem.

In considering the nature of many desirable generalizations of the problem, the minimization condition (2) is usually not fundamentally changed but the specification conditions (1) are generalized and/or expanded. It appears then that a proper first step in the order of indirection is in providing an alternative to the use of (2) while leaving the specifications (1) intact. 


\section{Lemmas for Reduced Matrix Methods}

There is an alternative to the use of (2). It is related to an orthogonality condition that, if $u_{i}$ and $v_{j}$ are constants subtracted from row $i$ and column $j$ respectively so that $c_{i j}-u_{i}-v_{j}=c_{i j}^{(t)} \geqq 0$ for all $i, j$ then the condition is

$$
x_{i j} c_{i j}^{(t)}=0 \quad i=1, \cdots, m ; j=1, \cdots, n .
$$

This means that $x_{i j} \neq 0$ only when $c_{i j}^{(t)}=0$. Now since every feasible primal solution which satisfies (3) has the smallest possible transformed sum, $\sum_{i, j} x_{i j} c_{i j}^{(t)}=0$, we have

Lemma 1: Any feasible solution for the primal problem (1) which satisfies (3) is an optimal solution for the primal problem (1) (2).

Any method which constructs the optimal primal solution with the use of the zero terms of the matrix $C^{(t)}$ of (3), rather than with the use of (2), may be called a reduced matrix method. If it also uses (1) without alternative conditions or theorems, the method may be said to be direct. There are reduced matrix methods which are indirect. See for example [5, 410] [8] [14] [10] which use such alternative results as Hall's condition [19], König-Egerváry Theorem [20], or Brogden's generalized condition [2] [8, 44].

More formally, since the smallest element of a set $S-k$, with $k$ constant corresponds to the smallest element of $S$, we have

Lemma 2: If $u_{i}$ is subtracted from row $i$ and $v_{j}$ from column $j$ for $i=1, \cdots, m$; $j=1, \cdots, n$, then $c_{i j}-u_{i}-v_{j}=c_{i j}^{(t)}$ results with

$$
\begin{array}{r}
x_{i j}^{(t)}=x_{i j}, \quad T^{(t)}=\sum_{i, j} x_{i j} c_{i j}^{(t)}=T-\sum_{i} a_{i} u_{i}-\sum_{j} b_{j} v_{j} \text { so } \\
T=T^{(t)}+\sum_{i} a_{i} u_{i}+\sum_{j} b_{j} v_{j} .
\end{array}
$$

Furthermore if $u_{i}$ and $v_{j}$ are chosen so that $c_{i j}^{(t)} \geqq 0$ and if $x_{i j}$ is taken $\neq 0$ only when $c_{i j}^{(t)}=0,(3)$ is satisfied, lemma 1 applies and $T^{(t)}=0$, with

$$
T=\sum_{i} a_{i} u_{i}+\sum_{j} b_{j} v_{j}
$$

Here $u_{i}$ and $v_{j}$ constitute a feasible solution for the dual; thus the direct method developed here is a dual algorithm.

\section{The Steps of the Reduction Process}

The direct method proceeds by a series of subtractions from rows and columns to produce a matrix $C^{(t)}$ with enough zero terms so that (3) and (1) are satisfied. These subtractions are of three different types and are presented in the three steps below.

Step 1. It is necessary that there be at least one zero term in each row and one in each column. The first step accomplishes this.

Determine

$$
v_{j}^{(0)}=\min _{i} c_{i j} \text { for } j=1, \cdots, n
$$

and write

$$
c_{i j}^{(0)}=c_{i j}-v_{j}^{(0)} \quad j=1, \cdots, n ; \quad i=1, \cdots, m .
$$


Then determine

$$
u_{i}^{(0)}=\min _{j} c_{i j}^{(0)} \quad \text { for } \quad i=1, \cdots, m
$$

and write

(5)

$$
c_{i j}^{(1)}=c_{i j}^{(0)}-u_{i}^{(0)}=c_{i j}-v_{j}^{(0)}-u_{i}^{(0)} \text { for all } i, j .
$$

TABLE 1

Balinski-Gomory Illustration [1]

\begin{tabular}{|c|c|c|c|c|c|}
\hline \multicolumn{6}{|c|}{$C^{(1)}$} \\
\hline$a_{i}^{b_{j}}$ & 2 & 2 & 3 & 4 & 4 \\
\hline 4 & & 4 & 1 & 0 & $0^{4}$ \\
\hline 5 & $0 y^{y+z-2}$ & 1 & $0^{3-z}$ & $0^{4-y}$ & 5 \\
\hline 6 & $0^{4-y-z}$ & $0^{2}$ & $0^{z}$ & $0^{y}$ & 1 \\
\hline
\end{tabular}

\begin{tabular}{|c|ccccc|c|}
\hline$a_{i}^{b j}$ & 2 & 2 & 3 & 4 & 4 & $y u_{i}^{(0)}$ \\
\hline 4 & 3 & 5 & 3 & 1 & 1 & \\
5 & 2 & 3 & 3 & 2 & 7 & 1 \\
6 & 1 & 1 & 2 & 1 & 2 & \\
\hline$v_{j}^{(0)}$ & 1 & 1 & 2 & 1 & 1 & 23 \\
\hline
\end{tabular}

Row-Column Analysis

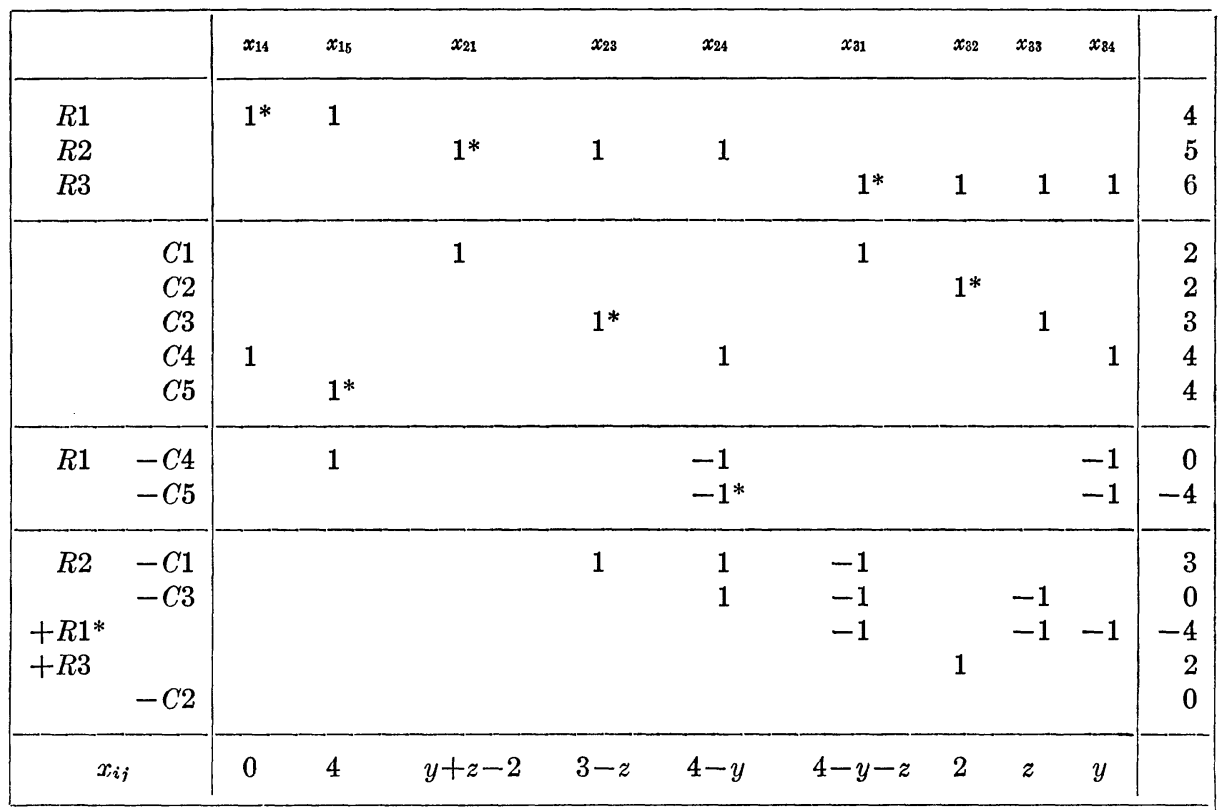

$0 \leqq y \leqq 4,0 \leqq z \leqq 3,2 \leqq y+z \leqq 4$ 
Then we have

$$
c_{i j}^{(1)} \geqq 0 \text { for all } i, j \text { and } c_{i j}^{(1)}=0
$$

for at least one element in each row and for one element in each column. This step is illustrated in Tables 1, 2, 3, and 6 where application is made to illustrative problems of earlier authors. The roles of column and row may be interchanged, if desired, with subtraction first from the row, as in Table 5. It should be noted that this first transformation is the equivalent of the first transformation [5, 405] of the Dantzig version of the Ford-Fulkerson primal-dual method [16, 95-111]. As the contribution to the bounding set sum, compute $S^{(0)}=\sum_{i} a_{i} u_{i}^{(0)}+$ $\sum_{j} b_{j} v_{j}^{(0)}$ and record it in the lower right box of the augmented matrix $C$. See Tables 1, 2, 3, 5, 6 .

In case there is a feasible solution to (1) which satisfies (3), the solution is complete and the matrix is said to be completely reduced. Thus $C^{(1)}$ is completely reduced in Table 1, but not in Tables 2, 3, 4, 5, 6. The determination of such a feasible solution is discussed in the section following. In Table 1 , the value of $T=S^{(0)}$ is placed in the lower right box of the matrix $C^{(1)}$.

For the existence of a feasible solution to (1) and (3) it is necessary that there be as many zero terms in each column (row), when weighted by the $a_{i}\left(b_{j}\right)$, as needed to meet the specification for that column (row). We then have Step 2. Let

$$
\delta_{i j}=\left\{\begin{array}{l}
1 \text { when } c_{i j}^{(1)}=0 \\
0 \text { otherwise }
\end{array}\right\} .
$$

Then with

it is necessary that

$$
g_{j}=\sum_{i=1}^{m} a_{i} \delta_{i j}
$$

$$
D_{j}=g_{j}-b_{j} \geqq 0 .
$$

If $D_{j}<0$ for some $j=J$, we speak of a deficiency in column $J$, i.e., a shortage of zero terms. To eliminate the deficiency in column $J$, or at least to make it smaller, we introduce additional zero terms. We determine, for the reduced matrix $C^{(r)}$ a number $\Delta$ defined by

$$
\Delta=\min _{i}\left\{c_{i J}^{(r)} \mid c_{i J}^{(r)}>0\right\}>0
$$

and define $I$ to be the subset of rows for which $c_{i J}^{(r)}=0$, with $\bar{J}$ and $\bar{I}$ the respective complement sets of $J$ and $I$. Then we subtract $\Delta$ from column $J$ and $-\Delta$ from all rows of $I$, to get

$$
c_{i j}^{(r+1)}=\left\{\begin{array}{c}
c_{i j}^{(r)}-\Delta \text { for all elements of } \bar{I} J \\
c_{i j}^{(r)}(=0) \text { for all elements of } I J \\
c_{i j}^{(r)} \geqq 0 \text { for all elements of } \bar{I} \bar{J} \\
c_{i j}^{(r)}+\Delta \text { for all elements of } I \bar{J}
\end{array}\right\} .
$$

Then

$$
c_{i j}^{(r+1)} \geqq 0, g_{J}^{(r+1)}>g_{J}^{(r)} \text { and }-D_{J}^{(r+1)}<-D_{J}^{(r)} \text { as desired. }
$$


TABLE 2

Dantzig Illustration. [4]

\begin{tabular}{|c|rrrrr|r|}
\hline \multicolumn{7}{c|}{$C$} \\
\hline$a_{i}^{b_{j}}$ & 3 & 3 & 3 & 2 & 2 & $u_{i}^{(0)}$ \\
\hline 1 & 3 & 2 & 1 & 2 & 3 & \\
5 & 5 & 4 & 3 & -1 & 1 & \\
7 & 0 & 2 & 3 & 4 & 5 & \\
\hline$v_{j}^{(0)}$ & 0 & 2 & 1 & -1 & 1 & 9 \\
\hline
\end{tabular}

\begin{tabular}{|r|rrrrr|r|r|}
\hline$a_{i}^{b_{j}}$ & 3 & 3 & 3 & 2 & 2 & $u_{i}^{(1)}$ & $D_{i}^{(1)}$ \\
\hline 1 & 3 & 0 & 0 & 3 & 2 & -2 & 5 \\
5 & 5 & 2 & 2 & 0 & 0 & & -1 \\
7 & 0 & 0 & 2 & 5 & 4 & & -1 \\
\hline$v_{j}^{(1)}$ & & & 2 & & & 4 & \\
\hline$D_{j}^{(1)}$ & 4 & 5 & $-2^{*}$ & 3 & 3 & & \\
\hline
\end{tabular}

\begin{tabular}{|c|ccccc|}
\hline$a_{i}^{b_{j}}$ & 3 & 3 & 3 & 2 & 2 \\
\hline 1 & 5 & 2 & $0^{1}$ & 5 & 4 \\
5 & 5 & 2 & $0^{1}$ & $0^{2}$ & $0^{2}$ \\
7 & $0^{3}$ & $0^{3}$ & $0^{1}$ & 5 & 4 \\
\hline
\end{tabular}

Row-Column Analysis

\begin{tabular}{|c|c|c|c|c|c|c|c|c|c|}
\hline & & $x_{13}$ & $x_{28}$ & $x_{24}$ & $x_{25}$ & $x_{31}$ & $x_{32}$ & $x_{33}$ & \\
\hline \multirow[t]{2}{*}{$\begin{array}{l}R 1 \\
R 2 \\
R 3\end{array}$} & & $1^{*}$ & $1^{*}$ & 1 & 1 & $1^{*}$ & 1 & 1 & $\begin{array}{l}1 \\
5 \\
7\end{array}$ \\
\hline & $\begin{array}{l}C 1 \\
C 2 \\
C 3 \\
C 4 \\
C 5\end{array}$ & 1 & 1 & $1^{*}$ & $1^{*}$ & 1 & $1^{*}$ & 1 & $\begin{array}{l}3 \\
3 \\
3 \\
2 \\
2\end{array}$ \\
\hline$R 3$ & $\begin{array}{l}-C 1 \\
-C 2\end{array}$ & & & & & & 1 & $\begin{array}{l}1 \\
1 *\end{array}$ & $\begin{array}{l}4 \\
1\end{array}$ \\
\hline \multirow[t]{2}{*}{$\begin{array}{r}R 1 \\
+R 2 \\
+R 3^{*}\end{array}$} & $\begin{array}{l}-C 3 \\
-C 4 \\
-C 5\end{array}$ & & -1 & 1 & $\begin{array}{l}1 \\
1\end{array}$ & & & $\begin{array}{l}-1 \\
-1 \\
-1 \\
-1\end{array}$ & $\begin{array}{r}-2 \\
3 \\
1 \\
-1 \\
0\end{array}$ \\
\hline & $x_{i j}$ & 1 & 1 & 2 & 2 & 3 & 3 & 1 & \\
\hline
\end{tabular}


TABLE 3

$A 4 \times 6$ Problem, $N=312$ [6]

\begin{tabular}{|c|c|c|c|c|c|c|c|c|}
\hline origin $i \backslash$ des & & $\begin{array}{l}\text { 号 } \\
\text { 煦 }\end{array}$ & 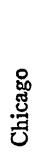 & 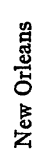 & 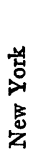 & $\begin{array}{l}\stackrel{n}{\vec{B}} \\
\dot{\Delta}\end{array}$ & 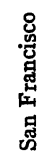 & \\
\hline & $a_{i}^{b j}$ & 48 & 11 & 32 & 92 & 50 & 79 & $u_{i}^{(0)}$ \\
\hline Boston & 82 & 5 & 9 & 16 & 2 & 12 & 32 & \\
\hline Cleveland & 88 & 2 & 3 & 11 & 5 & $\boldsymbol{5}$ & 26 & \\
\hline Kansas City & 99 & 10 & 6 & 8 & 12 & 3 & 19 & \\
\hline Los Angeles & 43 & 26 & 21 & 19 & 29 & 19 & 4 & \\
\hline & $v_{i}^{(0)}$ & 2 & 3 & 8 & 2 & 3 & 4 & 1035 \\
\hline
\end{tabular}

$C^{(1)}$

\begin{tabular}{|l|r|rrrrrr|r|r|r|}
\hline & $a_{i}^{b_{j}}$ & 48 & 11 & 32 & 92 & 50 & 79 & $u_{i}^{(1)}$ & \multicolumn{1}{|c|}{$D_{i}^{(1)}$} \\
\hline Boston & 82 & 3 & 6 & 8 & 0 & 9 & 28 & -3 & 10 \\
Cleveland & 88 & 0 & 0 & 3 & 3 & 2 & 22 & & -29 \\
Kansas City & 99 & 8 & 3 & 0 & 10 & 0 & 15 & & -17 \\
Los Angeles & 43 & 24 & 18 & 11 & 27 & 16 & 0 & -15 & 36 \\
\hline & $v_{j}^{(1)}$ & & & & 3 & & 15 & 570 & \\
\hline & $D_{j}^{(1)}$ & 40 & 77 & 67 & $-10^{* *}$ & 49 & $-36^{*}$ & & \\
\hline
\end{tabular}

$$
C^{(2)}
$$

\begin{tabular}{|l|r|rrrrrr|r|r|}
\hline & $a_{i}^{b_{j}}$ & 48 & 11 & 32 & 92 & 50 & 79 & $u_{i}^{(2)}$ & $D_{i}^{(2)}$ \\
\cline { 2 - 6 } & 82 & 6 & 9 & 11 & 0 & 12 & 16 & & 10 \\
Boston & 88 & 0 & 0 & 3 & 0 & 2 & 7 & & 63 \\
Cleveland & 99 & 8 & 3 & 0 & 7 & 0 & 0 & -2 & 62 \\
Kansas City & 43 & 39 & 33 & 26 & 39 & 31 & 0 & -2 & 36 \\
\hline & $v_{j}^{(2)}$ & & & 2 & & 2 & 2 & 38 & \\
\hline & $D_{j}^{(2)}$ & 40 & 77 & 67 & 78 & 49 & 63 & & \\
\hline
\end{tabular}

\begin{tabular}{|l|c|ccccccc|}
\hline & $C^{(3)}$ \\
\hline Boston & 48 & 11 & 32 & 92 & 50 & 79 \\
\hline Cleveland & 82 & 6 & 9 & 9 & $0^{82}$ & 10 & 14 \\
Kansas City & 88 & $0^{48}$ & $0^{11}$ & 1 & $0^{10}$ & $0^{19}$ & 5 \\
Los Angeles & 99 & 10 & 5 & $0^{32}$ & 9 & $0^{31}$ & $0^{36}$ \\
& 43 & 41 & 35 & 26 & 41 & 31 & $0^{43}$ \\
\hline
\end{tabular}


The contribution to the bounding set sum is

$$
S^{(r)}=\Delta b_{J}-\Delta g_{J}=\Delta\left(-D_{J}\right)=\Delta D^{\prime}{ }_{J}>0
$$

where $D_{J}^{\prime}=-D_{J}$.

Step 2 is first illustrated by application to the matrix $C^{(1)}$ of Table 2 where $J=3, g_{3}=1$ and $D_{J}=-2$. Then $I$ is row 1 and application of (9) results in the completely reduced matrix, $C^{(2)}$, since the unique solution, indicated by the

TABLE 3a

Row-Column Analysis for Matrix $C^{(2)}, N=312$ Problem

\begin{tabular}{|c|c|c|c|c|c|c|c|c|c|c|}
\hline & & $x_{14}$ & $x_{21}$ & $x_{22}$ & $x_{24}$ & $x_{33}$ & $x_{25}$ & $x_{36}$ & $x_{46}$ & \\
\hline $\begin{array}{l}R 1 \\
R 2 \\
R 3 \\
R 4\end{array}$ & & $1^{*}$ & $1^{*}$ & 1 & 1 & $1^{*}$ & 1 & 1 & $1^{*}$ & $\begin{array}{l}82 \\
88 \\
99 \\
43\end{array}$ \\
\hline & $\begin{array}{l}C 1 \\
C 2 \\
C 3 \\
C 4 \\
C 5 \\
C 6\end{array}$ & 1 & 1 & $1^{*}$ & 1 & 1 & $1^{*}$ & $1^{*}$ & 1 & $\begin{array}{l}48 \\
11 \\
32 \\
92 \\
50 \\
79\end{array}$ \\
\hline$R 1$ & $-C^{4}$ & & & & $1^{*}$ & & & & & -10 \\
\hline $\begin{array}{r}R 2 \\
+R 1^{*}\end{array}$ & $\begin{array}{l}-C 1 \\
-C 2\end{array}$ & & & 1 & $\begin{array}{l}1 \\
1\end{array}$ & & & & & $\begin{array}{l}40 \\
29 \\
19\end{array}$ \\
\hline $\begin{array}{r}R 3 \\
+R 4\end{array}$ & $\begin{array}{l}-C 3 \\
-C 5 \\
-C 6\end{array}$ & & & & & & 1 & $\begin{array}{l}1 \\
1\end{array}$ & -1 & $\begin{array}{r}67 \\
17 \\
-62 \\
-19\end{array}$ \\
\hline
\end{tabular}

TABLE $3 \mathrm{~b}$

Informal Analysis for Matrix $C^{(2)}, N=312$ Problem

$C^{(2)}$

\begin{tabular}{|c|c|c|c|c|c|c|c|c|}
\hline$a_{i}^{b_{j}}$ & 48 & 11 & 32 & 92 & 50 & 79 & $u_{i}^{(2)}$ & $A_{i}^{(2)}$ \\
\hline 82 & 6 & 9 & 11 & ${ }^{0} 0^{82}$ & 12 & 16 & 2 & ${ }^{10} 0$ \\
\hline 88 & 0048 & $0^{11}$ & 3 & $0^{10}$ & 2 & 7 & 2 & $1-19$ \\
\hline 99 & 8 & 3 & ${ }^{0} 0^{32}$ & 7 & $00^{50}$ & $0^{36}$ & & 219 \\
\hline 43 & 39 & 33 & 26 & 39 & 31 & ${ }^{0} 0^{43}$ & & ${ }^{2} 0$ \\
\hline$v_{j}^{(2)}$ & -2 & -2 & & -2 & & & 38 & \\
\hline$A_{j}^{(2)}$ & ${ }^{10} 0$ & ${ }^{1} 0$ & ${ }^{2} 0$ & ${ }^{10} 0$ & ${ }^{2} 0$ & ${ }^{2} 0$ & & 0 \\
\hline
\end{tabular}


right superscripts of the matrix, comes from the application of (1). Then $S^{(1)}=$ $2(3-1)=4$ and the value of $T$ is $S^{(0)}+S^{(1)}=9+4=13$ units as may be verified with the use of $T=\sum_{i, j} x_{i j} c_{i j}$.

Rows and columns may be interchanged, of course, to obtain values of $D_{i}$,

TABLE 4

Removing the Total Deficiency, Illustration of [10]

$C^{(1)}$

\begin{tabular}{|c|c|c|c|c|c|c|c|}
\hline$a_{a_{i}}^{b_{j}}$ & 4 & 1 & 4 & 3 & $u_{i}^{(1)}$ & $A_{i}^{(1)}$ & $\min$. \\
\hline 1 & $00^{1}$ & 1 & 5 & 6 & & 0 & 15 \\
\hline 1 & $00^{1}$ & 4 & 6 & 5 & & 0 & 15 \\
\hline 1 & $0^{11}$ & 8 & 2 & 1 & -2 & 0 & ${ }^{1} 1^{*}$ \\
\hline 1 & ${ }^{0} 0^{1}$ & 3 & 5 & 6 & & 0 & 15 \\
\hline 1 & 6 & 0 & 5 & 7 & & 0 & 15 \\
\hline 1 & 4 & 1 & $0^{y}$ & $0^{1-y}$ & -3 & 0 & 2 \\
\hline 1 & 2 & 3 & $0^{z}$ & $0^{1-z}$ & -3 & 0 & 2 \\
\hline 1 & 4 & 1 & $0^{0}$ & 2 & -3 & 0 & 2 \\
\hline 1 & 3 & 2 & $0^{u}$ & $0^{1-u}$ & -3 & 0 & 2 \\
\hline 1 & ${ }^{0} 0^{1}$ & 4 & 1 & 5 & -2 & 0 & ${ }^{1} 1^{*}$ \\
\hline 1 & 9 & 0 & 3 & 5 & & 0 & ${ }^{1} 3^{*}$ \\
\hline 1 & $0^{w}$ & $0^{1-w}$ & 7 & 7 & & 0 & 17 \\
\hline$v_{j}^{(1)}$ & 0 & 0 & 3 & 3 & 5 & & \\
\hline$A_{j}^{(1)}$ & ${ }^{11}+w$ & $12-w$ & ${ }^{2} y+z+u-3$ & ${ }^{2}-y-z-u$ & & 0 & \\
\hline
\end{tabular}

Formal analysis also shows $C 3+C 4-R 6-R 7-R 8-R 9=3$

\begin{tabular}{|c|cccc|}
\hline \multicolumn{5}{c|}{$C^{(2)}$} \\
\hline & 4 & 1 & 4 & \multicolumn{1}{c|}{3} \\
\hline 1 & $0^{1}$ & 1 & 2 & 3 \\
1 & $0^{1}$ & 4 & 3 & 2 \\
1 & 2 & 10 & 1 & $0^{1}$ \\
1 & $0^{1}$ & 3 & 2 & 3 \\
\hline 1 & 6 & 0 & 2 & 4 \\
1 & 7 & 4 & $0^{y}$ & $0^{1-y}$ \\
1 & 5 & 6 & $0^{z}$ & $0^{1-z}$ \\
1 & 5 & 4 & $0^{1}$ & 2 \\
\hline 1 & 6 & 5 & $0^{1-\gamma-z}$ & $0^{y^{+z}}$ \\
1 & 2 & 6 & $0^{1}$ & 4 \\
1 & 9 & $0^{0}$ & $0^{1}$ & 2 \\
1 & $0^{1}$ & $0^{0}$ & 4 & 4 \\
\hline
\end{tabular}

where $0 \leqq y \leqq 1$

$0 \leqq z \leqq 1$

$0 \leqq y+z \leqq 1$. 
as illustrated in Table 2. Then the negative $D_{i}$ or $D_{j}$ having largest absolute value is selected, as in Table 2 . Here the transformation which eliminates the deficiency in column 3 also eliminates the deficiencies in row 2 and row 3 . In general step 2 can be applied successively until all deficiencies in individual columns (rows) are removed. Frequently one can combine successive applications of step 2 into a single step with possible modification of the values to be subtracted from the rows and columns. Thus there are basically two applications of step 2 in going from $C^{(1)}$ to $C^{(2)}$ in Table 3. The second applies to column 4 , indicated by a double asterisk. In determining the $\Delta$ for the second application, we must use the minimum of the non-zero elements of column 4

TABLE 5

Negative Solution, Kuhn Illustration [21]

\begin{tabular}{|c|c|c|c|c|c|}
\hline$a_{i} b_{j}$ & 1 & 1 & 1 & 1 & $u_{i}^{(0)}$ \\
\hline 1 & 8 & 7 & 9 & 9 & 7 \\
\hline 1 & 5 & 2 & 7 & 8 & 2 \\
\hline 1 & 6 & 1 & 4 & 9 & 1 \\
\hline 1 & 2 & 3 & 2 & 6 & 2 \\
\hline$v_{j}^{(0)}$ & & & & 2 & 14 \\
\hline
\end{tabular}

\begin{tabular}{|c|c|c|c|c|c|}
\hline \multicolumn{6}{|c|}{$C^{(1)}$} \\
\hline$\widehat{a}_{i} b_{j}$ & 1 & 1 & 1 & 1 & $u_{i}^{(2)}$ \\
\hline 1 & 1 & 0 & 2 & 0 & \\
\hline 1 & 3 & 0 & 5 & 4 & \\
\hline 1 & 5 & 0 & 3 & 6 & \\
\hline 1 & 0 & 1 & 0 & 2 & -1 \\
\hline$v_{j}^{(2)}$ & 1 & & 1 & & 1 \\
\hline
\end{tabular}

$\begin{aligned} \rightarrow & \begin{array}{l}\text { Formal row-column analysis for } C^{(1)} \\ \text { shows } C 1+C 3-R 4=1 \\ \text { and } R 1+R 2+R 3-C 2-C 4=1\end{array} \\ & \text { Row column analysis for } C^{(2)}\end{aligned}$

\begin{tabular}{|c|c|c|c|c|c|}
\hline \multicolumn{6}{|c|}{$C^{(2)}$} \\
\hline$a_{i} b_{j}$ & 1 & 1 & 1 & 1 & $u_{i}^{(3)}$ \\
\hline 1 & $0^{1}$ & $0^{-1}$ & 1 & $0^{1}$ & \\
\hline 1 & 2 & $0^{1}$ & 4 & 4 & 2 \\
\hline 1 & 4 & $0^{1}$ & 2 & 6 & 2 \\
\hline 1 & $0^{0}$ & 2 & $0^{1}$ & 3 & \\
\hline$v_{j}^{(3)}$ & & -2 & & & 2 \\
\hline
\end{tabular}

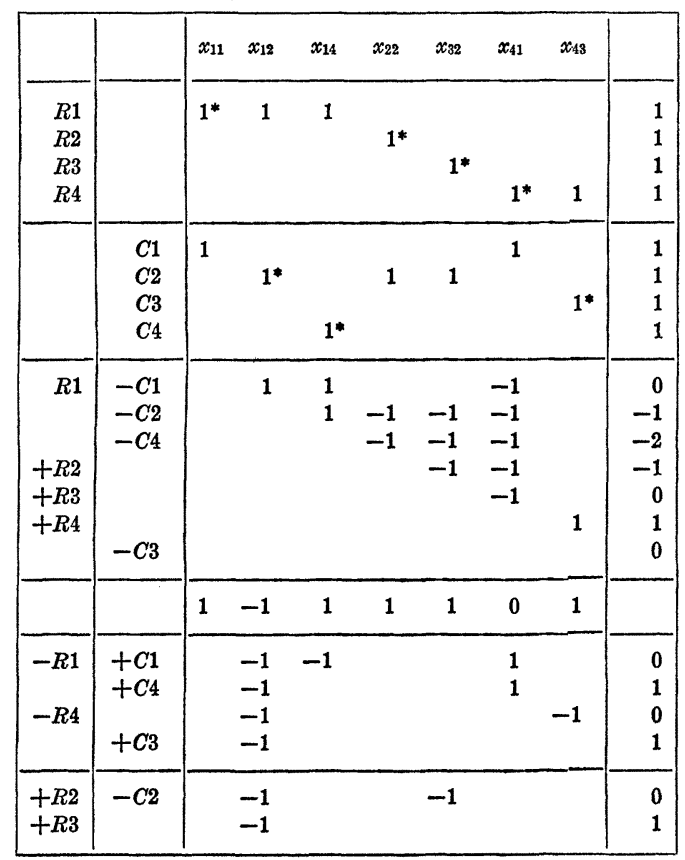

Use $C 1+C 3+C 4-R 1-R 4$ or $R 2+R 3-C 2$

$0 \leqq y \leqq 1$ 
resulting from the first application. For example in Table 3, the $\Delta$ for $J=4$, after application to $J=6$, is $\min (3,10,42)=3$ as indicated.

The use of step 2 is continued until a matrix is reached for which every $D_{j}$ and $D_{i} \geqq 0$. Such a matrix is said to be reduced grouped. The $C^{(2)}$ matrix of Table 3 is

TABLE 6

Problem with Variable Specifications, Charnes-Kirby Illustration [3]

$$
C
$$

\begin{tabular}{|l|ccrrr|c|}
\hline$a_{i} b_{j}$ & $11-\beta$ & 7 & 2 & 9 & 15 & $u_{i}^{(0)}$ \\
\hline 14 & 2 & 5 & -5 & 8 & 3 & \\
$20-\beta$ & 3 & 4 & 0 & 6 & 9 & \\
10 & 1 & 8 & 6 & 5 & -2 & \\
\hline$v_{j}^{(0)}$ & 1 & 4 & -5 & 5 & -2 & $44-\beta$ \\
\hline
\end{tabular}

$C^{(1)}$

\begin{tabular}{|l|rrrrr|r|r|}
\hline$a_{i} b_{j}$ & $11-\beta$ & 7 & 2 & 9 & 15 & $u_{i}^{(1)}$ & $D_{i}^{(1)}$ \\
\hline 14 & 1 & 1 & 0 & 3 & 5 & 1 & $-12^{*}$ \\
$20-\beta$ & 2 & 0 & 5 & 1 & 11 & & $\beta-13$ \\
10 & 0 & 4 & 11 & 0 & 0 & -4 & $25-\beta$ \\
\hline$v_{j}^{(1)}$ & & & -1 & 1 & 4 & 41 & \\
\hline$D_{j}^{(1)}$ & $\beta-1$ & $13-\beta$ & 12 & 1 & $-5^{* *}$ & & \\
\hline
\end{tabular}

$C^{(2)}$

\begin{tabular}{|l|ccccc|c|c|}
\hline$a_{i} b_{j}$ & $11-\beta$ & 7 & 2 & 9 & 15 & $u_{i}^{(2)}$ & $D_{i}^{(2)}$ \\
\hline 14 & $00^{11-\beta}$ & $0^{\beta-4}$ & $0^{2}$ & 1 & $0^{5}$ & & $21-\beta$ \\
$20-\beta$ & 2 & $0^{11-\beta}$ & 6 & $0^{9}$ & 7 & 2 & $\begin{array}{c}\beta-4^{*} \\
5\end{array}$ \\
\hline 10 & 4 & 8 & 16 & 3 & $00^{w}$ & & \\
\hline$v_{j}^{(2)}$ & & -2 & & -2 & & $8-2 \beta$ & \\
\hline$D_{i}^{(2)}$ & $3+\beta$ & $27-\beta$ & 12 & $11-\beta$ & 9 & & \\
\hline
\end{tabular}

$C^{(3)}$ with $0 \leqq \beta \leqq 4$

\begin{tabular}{|c|c|c|c|c|c|}
\hline$a_{i} b_{j}$ & $11-\beta$ & 7 & 2 & 9 & 15 \\
\hline 14 & $0^{7}$ & 2 & $0^{2}$ & 3 & $0^{5}$ \\
\hline $20-\beta$ & $0^{4-\beta}$ & $00^{7}$ & 4 & ${ }^{0} 0^{9}$ & 5 \\
\hline 10 & 4 & 10 & 16 & 5 & $0^{0} 10$ \\
\hline
\end{tabular}

Remarks

$0 \leqq \beta \leqq 11$

$\beta=11$ eliminates

first column.

no additional

restriction on $\beta$.

solution holds for

$4 \leqq \beta \leqq 11$ with

$T=85-\beta$.

solution holds for $0 \leqq \beta \leqq 4$ with $T=93-3 \beta$. 
reduced grouped as shown by the values of $D_{i}^{(2)}$ and $D_{j}^{(2)}$. But this matrix is not completely reduced since there is no feasible solution which satisfies (1) and (3). An attempt to provide such a solution starting with $x_{14}=82$ results in $x_{24}=10$, $x_{21}=48, x_{22}=11$ and since $x_{21}+x_{22}+x_{24}=69<88$, there is a deficiency of 19 units in rows 1 and 2. Alternately there is a deficiency in columns 3, 5, 6 which do not have zero terms in rows 1 or 2 . We then proceed to eliminate this deficiency by Step 3 .

For the existence of a feasible solution to (1) and (3), it is necessary that there be as many zero terms in each subset of columns (rows) as needed to meet the specifications for that subset of columns (rows). We then have Step 3.

Let a subset of columns be indicated by $J$ and let the subset of rows having at least one zero term in some column of $J$ be indicated by $I_{J}=I$. Then with $b_{J}=\sum_{j \varepsilon J} b_{j}$ and $g_{J}=\sum_{i \varepsilon I} a_{i}$, it is necessary that

$$
D_{J}=g_{J}-b_{J} \geqq 0 .
$$

If $D_{J}<0$ for some subset $J$, we speak of a deficiency in the columns of $J$, i.e., a shortage of zero terms with the deficiency $D^{\prime}{ }_{J}=-D_{J}>0$. Methods for determining such deficient subsets are discussed in the section following. In the Matrix $C^{(2)}$ of Table 3, we see $J=\{3,5,6\}$ is deficient since $I=\{3,4\}$ and $D_{J}=142-161=-19$. To eliminate this deficiency, or at least to make it smaller, we introduce additional zero terms. We determine, for a matrix $C^{(r)}$

$$
\Delta=\min c_{\bar{I} J}^{(r)}=\min \left\{\left.c_{i j}^{(r)}\right|_{i \varepsilon \bar{I}}, j_{\varepsilon} J\right\}>0
$$

and subtract $\Delta$ from all columns of $J,-\Delta$ from all rows of $I$ to obtain (9), which here holds for more general $J$ except that $c_{i j}^{(r)}=0$ for $I J$ is modified to $c_{i j}^{(r)} \geqq 0$. Then (10) and (11) also hold.

Step 3 is illustrated in the matrix $C^{(2)}$ of Table 3 where $\Delta=c_{25}^{(2)}=2$. The contribution to the bounding set sum is $S^{(2)}=2(19)=38$ units.

There are corresponding results when column and row are interchanged. Thus the subtraction of 2 units from rows 1 and $2,-2$ units from columns $1,2,4$, lead to the same $C^{(3)}$.

Application of step 3 is continued until the matrix is completely reduced with no subset of columns (rows) deficient.

\section{The Reduction Process}

In a sense these three steps are the building blocks of the reduction process. In this sense the specifications (1) provide the direction for the reduction process and the mortar needed for a unified structure. As might be expected with a direct method, the essence of the process is simple. Using lemma 1, either a solution results immediately or, if not, a deficiency is discovered which indicates the next step. There is an informal version of the process for hand calculation and a formal version for machine calculation.

Using the values of $a_{i}$ and $b_{j}$ shown in the matrix $C^{(2)}$ of Table 2 , it is seen that the superscripts, indicating the values of $x_{i j} \neq 0$, can be assigned at once. Similarly this can be done in the matrix $C^{(1)}$ of Table 1 , but here there are multiple 
solutions so parameters, $y$ and $z$, are used to indicate multiple choices after all unique assignments are first made. The result is a general (parametric) solution. For the usual restricted problem, the values of the parameters producing negative and non-integral solutions must be eliminated. Thus in Table 1, admissible values are integral values satisfying $0 \leqq y \leqq 4,0 \leqq z \leqq 3$, subject to $2 \leqq y+$ $z \leqq 4$. The solutions are $(y, z)=(0,2)(0,3)(1,1)(1,2)(1,3)(2,0)(2,1)$ $(2,2)(3,0)(3,1)(4,0)$.

A more formal reduction process, suitable for machine programs, is presented in the row-column analysis. We write the value of $x_{i j}$ associated with $c_{i j}^{(t)}=0$ terms as the headings for columns. We then write the equation coefficients for each row and each column. We wish to know if these equations are consistent. We reduce these equations essentially to triangular form by marking (with an asterisk) the unit coefficient at the left of each equation subject to the condition that no column can be marked more than once. We then take in turn each equation which has no marked term, eliminate terms by adding to or subtracting from marked equations, until the left hand element appears in an unmarked column (in which case the element is marked) or until all units on the left side of the equation are eliminated. In case all the corresponding elements on the right are zero, the equations are consistent and the back solution proceeds as is shown in Tables 1 and 2. In the back solution a parameter value, $y, z, \cdots$, is assigned to each unmarked column. A general parametric solution results.

In Table 1 and in Table 2, the formal row-column analysis leads to the solution. The matrix is completely reduced. The value of the minimum $T$ is the net sum of the $S^{(r)}$ (as recorded in the right lower box for each matrix) and can be verified by applying the $x_{i j}$ values to the original $c_{i j}$.

The notation in the row-column analysis needs some explanation. Formally the notation $R i$ means $\sum_{j=1}^{n} x_{i j}=a_{i}$ for fixed $i$ and $C j$ means $\sum_{i=1}^{m} x_{i j}=b_{j}$ for fixed $j$. But since $x_{i j}=0$ when $c_{i j}^{(t)} \neq 0$, we may omit all these terms. Thus in Table $2, R 3^{*}=R 3-C 1-C 2$ is $\left(x_{31}+x_{32}+x_{33}=7\right)-\left(x_{31}=3\right)-\left(x_{32}=3\right)$ and is $x_{33}=1$ as indicated in Table 2. Furthermore the result $R 1+R 2+R 3^{*}-$ $C 3-C 4-C 5$, when the value of $R 3^{*}$ is inserted, indicates that every $x_{i j}$ is multiplied by +1 for row $i$ and by -1 for column $j$ to give a net result of zero.

The formal version is also illustrated in Table 3 and Table 3a. This problem is a realistic problem with the matrix $C$ consisting of distances (in 100 miles) between origins and destinations. Applications of step 1 and step 2 result in the matrix $C^{(2)}$. Row-column analysis for the matrix $C^{(2)}$ of Table 3a shows complementary inconsistencies so that either of the complementary forms $R 1+R 2-$ $C 1-C 2-C 4=19$ or $C 3+C 5+C 6-R 3-R 4=19$ can be used. Using the second we subtract $\Delta$ from $C 3, C 5$, and $C 6$ and $-\Delta$ from $R 3$ and $R 4$ with a net subtraction of $19 \Delta$. From the matrix $C^{(2)}$ we see that $\Delta=c_{25}^{(2)}=2$ is the smallest value in $\bar{I} J$. The transformation with $\Delta=2$ is made and produces the completely reduced matrix $C^{(2)}$ with the values of $x_{i j}$, and the value of $T$, as indicated.

In more complex problems there may be many, say $k$, inconsistencies. However there are only $k-1$ independent ones since it is always true that $\sum_{i=1}^{m} R i-$ 
$\sum_{j=1}^{n} C j=0$. One plan is to select the transformation associated with the largest deficiency. If one wishes he may determine the value of $\Delta$ for each inconsistency and hence select the transformation which maximizes $S^{(r)}=D^{\prime} \Delta$ at each step. However the reduction process proceeds satisfactorily with the elimination of any deficiency.

Though formal row-column analysis is available for machine calculation, easy and informal analysis based on (1), can be used with hand calculation. The organization of the informal version is shown in Table $3 \mathrm{~b}$. The tentative $x_{i j}$ assignments are made first for all rows and columns with a single zero entry. For later identification these are marked with a left upper superscript ${ }^{0}$. The assignments are then made by columns, in accordance with conditions (1), with parameters $y, z, \cdots$ used when the assignment is not unique with the values of $A_{j}$ and $A_{i}$, the differences between the assigned and quota values, inserted in the last row and column. Here there is a deficiency of 19 in row 2 and a surplus of 19 in row 3. The rows and columns which are involved in the respective linear forms are indicated by the left superscripts 1 and 2 in the $A_{j}$ row and $A_{i}$ column. Thus the first linear form involves $R 1, R 2, C 1, C 2, C 4$. The second linear form is here the complement of the first. These, of course, are the identical linear forms resulting from the formal row-column analysis of Table 3a. Using the rows and columns of the first linear form, there is a deficiency of 19 as shown in row 2 . Then $\Delta=2$ and the subtractions are as indicated. The resulting matrix $C^{(3)}$ is completely reduced as it has the unique solution indicated in Table 3.

\section{Removing the Total Deficiency}

If there is only one element of $\bar{I} J$ equal to $\Delta$ and if either of the corresponding $a_{i}$ or $b_{j}$ is not as large as the deficiency $D^{\prime}$, the transformation does not completely remove the deficiency though, of course, the whole process can be repeated. More compactly if the columns (rows) are deficient, these repetitive steps can be combined in a single step with the largest of the (weighted) $D^{\prime}$ row (column) minima used as $\Delta$, followed by the appropriate negative subtractions to introduce the required number of zero terms in $\bar{I} J$. The essential requirements of a reduced matrix method are met though some zero terms may become positive.

An illustration is presented in Table 4. Initial transformations result in a matrix $C^{(1)}$ with a net subtraction of 101 units. Formal row-column analysis (not presented) shows the inconsistency $C 3+C 4-R 6-R 7-R 8-R 9=3$ while the informal version of Table 4 shows at once a deficiency of 3 in $J=$ $\{3,4\}$ with $I=\{6,7,8,9\}$. Since all $a_{i}=1$, subtraction of $\Delta=1$, the smallest non-zero element in $\bar{I} J$, which appears here in two different rows, results in the removal of but two units of the deficiency. To remove the full deficiency at one step, we record the minimum in $J$ for each row of $\bar{I} J$ as shown in the last column of the matrix $C^{(1)}$ of Table 4. The third largest minimum, 3 , is subtracted from $J$ and $0 \leqq \epsilon_{i} \leqq 3$ are subtracted from the appropriate rows to preserve the form of the reduced matrix. The resulting matrix $C^{(2)}$ is completely reduced having 3 solutions indicated by $(y, z)=(0,0)(0,1)(1,0)$. 


\section{Negative Solution Transformations}

The formal process continues until no deficiencies remain. However since there is no specification, explicit or implicit, that $x_{i j} \geqq 0$ or that $x_{i j}$ is integral, the resulting parametric solution is a general solution with no restrictions on the $x_{i j}$. The more restricted form of the problem, as stated above, requires a solution with integral $x_{i j} \geqq 0$. To obtain a solution for such a restricted problem from a general solution in wbich one or more $x_{i j}$ may be negative, it is appropriate to introduce a transformation to eliminate the negative solution. Such a transformation is called a negative solution transformation.

Consider, for example, an example of Kuhn [21], as shown in Table 5. The formal row-column analysis for $C^{(2)}$ shows no inconsistencies but the resulting general solution shows $x_{12}=-1$. More generally we consider an elimination of the negative result $x_{i j}=-B$, with $B>0$. Then, a linear form of the equations (1) results in $x_{i j}=-B$. To obtain the explicit value of such a form it is only necessary to take some equation of (1) containing the negative $x_{i j}$, multiply it by -1 , and eliminate all the other $x_{i j}$ terms to get a linear form of $I$ and $J$ which can be used as the basis of the desired transformation. If, as before, we subtract $\Delta$ from the rows (columns) having plus signs in the form and $-\Delta$ from the columns (rows) having minus signs, the net amount of the subtraction is $\Delta B$, the amount of the increment to the bounding set sum. At least one new zero term is introduced and the zero term in the $i, j$ position is replaced by $\Delta$ so the negative solution is eliminated. Thus in the row-column analysis for the matrix $C^{(2)}$ of Table 5, once it is established that $x_{12}=-1,-R 1$ is selected and the other terms are eliminated. The resulting form is $C 1+C 3+C 4-R 1-R 4$ which is $-x_{12}=1$. Alternately the selection of $-C 2$ leads to $R 2+R 3-C 2$ which is $-x_{12}=1$. Using $R 2+R 3-C 2$ we see that the smallest element in $\bar{I} J$ is $c_{33}^{(3)}=2$ so $\Delta=2$ units are subtracted from rows 2 and 3 and -2 units from column 2 with a net subtraction of $2(2)-2(1)=2$ units. The resultant matrix $C^{(4)}$ is completely reduced and has the solutions indicated in Table 5 with integral $y=0$ or $y=1$.

The formal version of the method is useful in obtaining general answers, including the possibility of solution sets with negative numbers, and in obtaining answers to more general problems such as the $k$-dimensional transportation problem as shown below. For the more specific two-dimensional problem, conditions which implicitly require $x_{i j} \geqq 0$ can be used in the less formal version to avoid negative $x_{i j}$, if a solution to the restricted problem is preferred.

\section{Avoidance of Negative Solutions}

In the transportation problem with $a_{i}$ and $b_{j}$ of some size and not all the same, the formal method results infrequently in negative solutions. Negative $x_{i j}$ do occur sometimes in the solution, of the assignment problem $\left(a_{i}=b_{j}=1\right)$ as is illustrated in Table 5 where it is necessary that $x_{12}=-1$ to cancel off one of the surplus values in row 1 and one of those in column 2 . For the transportation generalization of the problem of Table 5 with $b_{3}+b_{2} \geqq a_{4}$ the negative solution results only when $a_{1}-b_{1}-b_{4}$ equals $b_{2}-a_{2}-a_{3}$ and is negative. 
Negative solutions can be avoided with the determination and elimination of surplus assignments. When negative assignments are not permitted, the preliminary transformations may lead to reduced matrices with more assignments to some column (row) than specified by the quotas. The determination of such a surplus also leads to the determination of a transformation. Thus in the matrix $C^{(1)}$ of Table 5 we see that the initial assignments leads to a surplus of one unit in column 2 , and to a surplus of one unit in row 4 . Considering the surplus in column 2 , there must be an additional zero term provided in row 2 or row 3 . Here $\Delta=3$, and the subtraction of 3 units from row 2 and row 3, with the subtraction of -3 units from column 2 , leads to an increment to the bounding set sum of 3 units and to the completely reduced matrix $C^{(3)}$. In this case the surplus in column 2, and the surplus in row 4 , are fortunately removed at one step. The simple removal of the surplus in row 4 with $\Delta=1$ does not result in the removal of the surplus in column 2. An additional transformation with $\Delta=2$ is needed, as in the formal version shown in Table 5 .

In general let $J$ be a column with a surplus and $I$ the set of rows containing the non-negative assigned elements of $J$. Then we use the minimum of $\bar{I} J$ to determine $\Delta$ and subtract $\Delta$ from $I,-\Delta$ from $J$.

Accomplishing somewhat the same objective as the transformation above is the compression subroutine of Flood [14, 261].

With this device for avoiding negative solutions, the techniques of the informal version seldom lead to a negative solution. If they should, one can always eliminate the negative solution.

\section{Use of Rectangular Subgraphs}

It may be helpful in understanding the formal and informal versions to use the concept of linear subgraph. Consider the lines connecting the zero terms in the rows and columns of the reduced matrices. Linear subgraphs are identified by the results of the row-column subset analysis. Thus the matrix $C^{(2)}$ of Table 3 has one linear subgraph consisting of $R 1, R 2, C 1, C 2, C 4$ and a complementary one of $R 3, R 4, C 3, C 5, C 6$.

If $D^{\prime}=0$ for each subgraph, then $\Sigma R i=\Sigma C j$ for that subgraph and the subgraphs are rectangular. It is only with rectangular subgraphs that a solution exists though some components of the solution may be negative. If $\Sigma R i=\Sigma C j$ for all subgraphs, a general solution exists.

Each transformation introduces at least one additional zero so that at least two subgraphs are joined and the number of subgraphs is reduced by at least one at each step. Starting with a reduced matrix and with $n \leqq m$, it is seen at once that there can not be more than $n$ linear subgraphs. With reduced grouped matrices, the number is usually much less than this. Then the reduction to two complementary subgraphs can be made in no more than $n-2$ transformations, once the matrix is reduced. With the informal version, the number of linear subgraphs once the reduced grouped matrices have been further reduced with the use of transformations eliminating surplus assignments, is usually very small. 


\section{Number of Steps}

The direct method using reduced matrices, either formal or informal, is in a real sense not an iterative method at all like the simplex method. Rather a solution process is used in which the technique employed depends on the amount of reduction present.

The reduced matrix can be computed in one step. The reduced grouped matrix can then be found in at least $(m+n)$ steps (many of which can be integrated into a single step). With $m \geqq n$, and eliminating the total deficiency of a linear form in one step, the linear forms can be integrated in at most $n-2$ steps so a bound for the number of steps of the general solution is $m+2 n-1$. No problem is known for which the actual number of steps is more than a small proportion of this.

In the restricted problem, with integral $x_{i j} \geqq 0$, additional steps remove the negative solutions (formal) or the surplus assignment transformations (informal). There are usually very few of these. It is difficult to put an absolute bound on the number of steps needed to eliminate all negative solutions since the elimination of one negative $x_{i j}$ may result in the introduction of other negative $x_{i j}$ but $m+n$ seems to be a very adequate, though not absolutely guaranteed, bound for the number of these steps. Commonly for transportation problems with unequal $a_{i}$ and $b_{j}$ of some magnitude, no such transformations are needed.

\section{Solution of More General Problems}

The formal version of this direct method with reduced matrices is directly applicable to many generalizations of the transportation problem in which the conditions (1) are replaced by a more general set of conditions. It seems proper to mention some of these problems here and to give more adequate treatment in other papers. In all of these problems the importance of the use of completely reduced matrices as a substitute for (2) cannot be overemphasized.

\section{Problems with General $a_{i}$ and $b_{j}$}

There are many different $c_{i j}$ matrices that have the same completely reduced matrix and there are commonly many values of $a_{i}$ and $b_{j}$ appropriate to a given completely reduced matrix. Once a completely reduced matrix is available, it is a relatively trivial matter to write out the general solution for general $a_{i}>0$ and $b_{j}>0$. Thus we see at once, from the completely reduced matrix $C^{(2)}$ of Table 2 , that $x_{13}=a_{1}, x_{25}=b_{5}, x_{24}=b_{4}, x_{23}=a_{2}-b_{4}-b_{5}, x_{31}=b_{1}, x_{32}=b_{2}$ and $x_{33}=a_{3}-b_{1}-b_{2}$ provided only that $a_{2} \geqq b_{4}+b_{5}$ and $a_{3} \geqq b_{1}+b_{2}$. This is the general solution for any problem having matrix $c_{i j}^{\prime}=c_{i j}+\epsilon_{i}+\epsilon_{j}$ where $-\infty<\epsilon_{i}<\infty,-\infty<\epsilon_{j}<\infty$ for $i=1, \cdots, m$ and $j=1, \cdots, n$.

The solution of the general problem, $a_{i}>0, b_{j}>0$ when the specific problem has a parametric solution is next illustrated. From the matrix $C^{(1)}$ of Table 1 we see that $x_{34}=y, x_{33}=z, x_{32}=b_{2}, x_{31}=a_{3}-b_{2}-y-z, x_{24}=b_{4}+b_{5}-$ $a_{1}-y, x_{23}=b_{3}-z, x_{21}=b_{1}+b_{2}-a_{3}+y+z, x_{15}=b_{5}$ and $x_{14}=a_{1}-b_{5}$ as long as $a_{1} \geqq b_{5}, a_{3} \geqq b_{2}, b_{1}+b_{2} \geqq a_{3}, b_{4}+b_{5} \geqq a_{1}$ for non-negative $z$ and $y$ 
which satisfy $0 \leqq z \leqq b_{3}, 0 \leqq y \leqq b_{4}+b_{5}-a_{1}$ and $b_{1}+b_{2}-a_{3} \leqq y+z \leqq$ $a_{3}-b_{2}$.

Some matrices which are not completely reduced may also be used to give solutions to more general problems with $x_{i j} \geqq 0$. Thus the matrix $C^{(1)}$ of Table 5 with $a_{i}>0$ and $b_{j}>0$ is a completely reduced matrix for any problem with $b_{2} \geqq a_{2}+a_{3}$ and $a_{4} \geqq b_{1}+b_{2}$ while the $C^{(2)}$ matrix is completely reduced for any problem with $b_{2} \geqq a_{2}+a_{3}$ and $b_{3} \leqq a_{4} \leqq b_{1}+b_{3}$.

It is useful to note that any negative solution of the problem with fixed specifications corresponds to a non-negative solution of a problem with $a_{i}$ and $b_{j}$ increased by at least the amount of the negative solution. Thus the matrix $C^{(3)}$ of Table 5 is a completely reduced matrix for all problems with specifications $a_{i}=1+\beta, 1,1,1 ; b_{j}=1,1+\beta, 1,1$ for all positive integral values of $\beta$ and for all matrices which reduce to $C^{(3)}$.

\section{Problems with One $a_{i}$ and One $b_{j}$ General}

Not quite so general but still useful is the case in which one $a_{i}$ becomes $a_{i} \pm \beta$ and some $b_{j}$ becomes $b_{j} \pm \beta$. The method proceeds as before. It is only necessary to avoid deficiencies and negative values of $x_{i j}$. As an illustration, a problem of Charnes-Kirby [3] is used in which $a_{2}=20$ is replaced by $20-\beta$ and $b_{1}=11$ is replaced by $11-\beta$. The solution is given in Table 6 using the informal version. The steps are routine and remarks interpret the results. There is a deficiency in row 2 of the matrix $C^{(2)}$ when $\beta<4$ but the resulting transformation yields the matrix $C^{(3)}$ which is completely reduced for $0 \leqq \beta \leqq 4$. The solution for integral non-negative $\beta$ requires no more steps than does the solution for $\beta=0$.

As a more practical and explicit illustration consider the problem of Table 3. Suppose facilities at Los Angeles are increased by 20 units and deliveries at San Francisco are increased by the same amount. Then the solution is immediately obtained from the completely reduced matrix of Table 3 with $x_{46}$ increased from 43 to 63 . If instead the deliveries at St. Louis are increased by 20 units, the matrix $C^{(3)}$ again provides the solution with $x_{46}=63, x_{36}=16, x_{33}=51$ and the other $x_{i j}$ not changed.

\section{Problems with Inadmissible Squares}

Problems with inadmissible squares are very easy to handle with the direct method using reduced matrices for solutions with $x_{i j} \geqq 0$. It is only necessary to fix $x_{i j}=0$ for every inadmissible square. This can be accomplished by making the $c_{i j}$ for each inadmissible square so large that the subtractions of the reduction process never reduce it to zero. Operationally this can be managed by placing an $X$ in each inadmissible square and using an $X$ in the next matrix when a constant is subtracted. Of course there may be so many inadmissible squares that no solution is possible but this method of reduced matrices then reveals the fundamental inconsistency since eventually the reduction process leads to some $\Delta=X$ which can not be used in a transformation. 


\section{Bounded Variable Transportation Problems}

Completely reduced matrices are useful in solving bounded variable transportation problems since the relations involving the bounded variables are usually expressed as conditions additional to (1). Solutions are available for types of problems which the $x_{i j}$ are bounded $[5,378]$ and in which partial sums of the $x_{i j}$ are bounded [15]. It is planned to discuss these results in a separate paper.

\section{Constant Fixed Charge Transportation Problems}

The general solutions are useful in solving fixed charge transportation problems with constant fixed charges. Once a general parametric solution is available, as it is with the direct method using reduced matrices, it is only necessary to determine the parameters so as to wipe out as many $x_{i j}$ terms as possible. Thus in Table 1, the optimal constant fixed charge solution is given by $z=0, y=4$ since $x_{24}, x_{31}$, and $x_{34}$ are wiped out and no other admissible values of $y$ and $z$ can wipe out so many $x$ 's. A paper on this subject has been accepted by The Naval Research Logistics Quarterly for the September 1966 issue [11].

\section{The General Transportation Problem}

The $k$-dimensional transportation problem, with specified capacities at intermediate points, etc. can be solved with the formal version of the direct method, though in this case fractional solutions as well as negative solutions may be encountered. Fractional solution transformations are similar to negative solution transformations. This problem is the mathematical equivalent of the group assembly problem [7]. Earlier versions of solutions are available in the literature and B. A. Galler has prepared a machine program for problems with as many dimensions as 20 [9] and [17]. The direct generality of the formal method is seen from the fact the program in the $k$ dimensions is also designed for the $k=2$ dimensional problem under consideration in this paper.

\section{Conclusion}

The direct method using reduced matrices is applicable to many direct generalizations of the basic problem. However an important claim for its use in the Hitchcock problem is its simplicity and ease and the speed, as compared with available alternative methods, particularly for the informal version, with which a general solution can be reached.

\section{Acknowledgment}

Improvements in the final version have resulted from conversations with Robert M. Thrall, with Merrill M. Flood, with John M. Dwyer, and from the suggestions of the referee.

\section{References}

1. Balinski, M. S. AND Gomory, R. E., "A Primal Method for the Assignment and Transportation Problems," Management Science, Vol. 10 (1964), 578-593. 
2. Brogden, H. E., "An Approach to the Problem of Differential Prediction," Psychometrika, Vol. 11 (1946), 139-154.

3. Charnes, A., And Kirby, M., "The Dual Method and the Method of Balas and Ivanescu for the Transportation Model," Cahièrs du Centre d'Etudes de Récherche Operationnel, Vol. 6 (1964), 5-18.

4. Dantzig, G. B., "Application of the Simplex Method to a Transportation Problem," Activity Analysis of Production and Allocation, T. C. Koopmans (ed.), New York, Wiley, 1951, 359-373.

5. - - Linear Programming and Extensions. Princeton, Princeton University Press, 1963.

6. Dwyer, P. S., "The Solution of the Hitchcock Transportation Problem with a Method of Reduced Matrices," University of Michigan, Dec. 1955 (hectographed).

7. - " "The Problem of Optimal Group Assembly," Symposium on Air Force Human Engineering, Personnel, and Training Research, G. Finch and F. Cameron (ed.), Air Research and Development Command, Baltimore, 1956, 104-114.

8. —-, "The Detailed Method of Optimal Regions," Psychometrika, Vol. 22 (1957), 4352 .

9. - - AND Galler, B. A., "The Method of Reduced Matrices for a General Transportation Problem," Journal of the Association for Computing Machinery, Vol. 4 (1957), 308313.

10. —, AND —-, "A Method for the Solution of Transportation Problems with Tall Matrices," in preparation.

11. - - "Use of Completely Reduced Matrices in Solving Transportation Problems with Fixed Charges," to appear in Naval Research Logistics Quarterly, September 1966.

12. Easterfield, T. E., "A Combinatorial Algorithm," Journal London Math. Society, Vol. 21 (1946), 219-226.

13. Flood, M. M., "On The Hitchcock Distribution Problem," Pacific Journal of Mathematics, Vol. 3 (1953), 369-386.

14. - " "A Transportation Algorithm and Code," Naval Research Logistics Quarterly, Vol. $8^{\circ}$ (1961), 257-276.

15. Ford, L. R. And Fulkerson, D. R., "A Primal-Dual Algorithm for the Capacitated Hitchcock Problem," Naval Research Logistics Quarterly, Vol. 4 (1957), 47-54.

16. —, AND —, "Flows in Networks," Princeton, Princeton University Press, 1962.

17. Galler, B. A. and Dwyer, P. S., "Translating the Method of Reduced Matrices to Machines," Naval Research Logistics Quarterly, Vol. 4 (1957), 55-71.

18. Glemzal, A., "An Algorithm for Solving the Transportation Problem," Journal of Research of Nat. Bur. Stand., Vol. 54 (1955), 213-216.

19. Hali, P., “On Representatives of Subsets," Journal London Math. Society, Vol. 10 (1935), 26-30.

20. KöNIG, D., "Über Graphen und ihre Anwendung auf Determintentheorie und Mengenlehre," Math. Ann. Vol. 77 (1916), 453-465.

21. KuHN, H. W., "The Hungarian Method for the Assignment Problem," Naval Research Logistics Quarterly, Vol. 2 (1955), 83-93.

22. - AND Baumol, M., "An Approximate Algorithm for the Fixed Charges Transportation Problem," Naval Research Logistics Quarterly, Vol. 9 (1962), 1-15. 